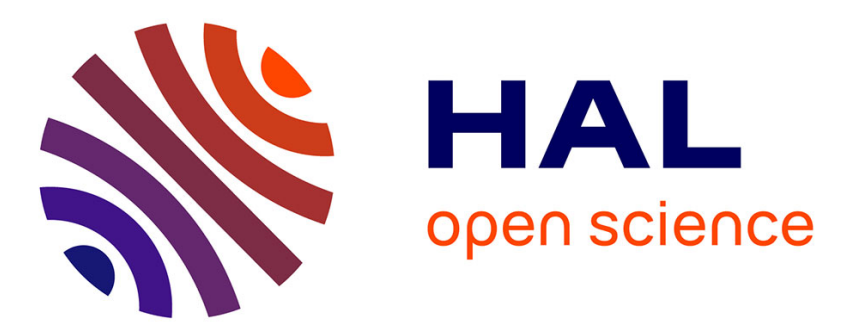

\title{
Estimation precision of the degree of linear polarization and of the angle of polarization in the presence of different sources of noise
}

François Goudail, Arnaud Bénière

\section{- To cite this version:}

François Goudail, Arnaud Bénière. Estimation precision of the degree of linear polarization and of the angle of polarization in the presence of different sources of noise. Applied optics, 2010, 49 (4), pp.683-693. hal-00747047

\section{HAL Id: hal-00747047 \\ https://hal-iogs.archives-ouvertes.fr/hal-00747047}

Submitted on 30 Oct 2012

HAL is a multi-disciplinary open access archive for the deposit and dissemination of scientific research documents, whether they are published or not. The documents may come from teaching and research institutions in France or abroad, or from public or private research centers.
L'archive ouverte pluridisciplinaire $\mathbf{H A L}$, est destinée au dépôt et à la diffusion de documents scientifiques de niveau recherche, publiés ou non, émanant des établissements d'enseignement et de recherche français ou étrangers, des laboratoires publics ou privés. 


\title{
Estimation precision of the degree of linear polarization and of the angle of polarization in the presence of different sources of noise
}

\author{
François Goudail* and Arnaud Bénière \\ Laboratoire Charles Fabry de l'Institut d'Optique, Centre National de la Recherche Scientifique, \\ Université Paris-Sud, Campus Polytechnique, 128, 91127 Palaiseau, France \\ *Corresponding author: francois.goudail @institutoptique.fr \\ Received 13 October 2009; accepted 17 November 2009; \\ posted 23 December 2009 (Doc. ID 118473); published 27 January 2010
}

\begin{abstract}
We consider imaging systems that measure the three first elements of the Stokes vector and deduce from them the degree of linear polarization and the angle of polarization. They require the acquisition of at least three intensity measurements, but performing more measurements is often thought to improve the estimation precision. We show that if the total acquisition time is fixed, the optimal number of measurements depends on the type of noise that affects the image: the estimation variance increases with the number of measurements $N$ when the noise is additive; it is independent of $N$ in the presence of Poisson shot noise and decreases with $N$ when the angles of the analyzers fluctuate. In general, the optimal number of measurements results from a compromise on the robustness of these different types of perturbations. (C) 2010 Optical Society of America

OCIS codes: $\quad 260.5430,030.4280$.
\end{abstract}

\section{Introduction}

In many cases, the circular contribution to polarized light reflected by materials can be considered negligible, and measuring the three first elements of the Stokes vector is sufficient. It is, in particular, the case when measuring images of outdoor scenes illuminated by unpolarized natural sources. The polarization features of the backscattered light mainly arise from Fresnel-like reflection on facets with different orientations, and this phenomenon produces light that is partially polarized but whose principal polarization state [1] is linear. In this case, the last element of the Stokes vector, which corresponds to ellipticity, can be considered null with good approximation [1]. The Stokes vector limited to the three first components is classically named linear Stokes vector. From its measurement, two main parameters

0003-6935/10/040683-11\$15.00/0

(C) 2010 Optical Society of America can be estimated: the degree of linear polarization (DOLP) and the angle of the principal state of polarization, which we will call the angle of polarization (AOP). These two parameters are used, for example, for estimating the absolute orientation of facets in a scene in order to reconstruct it in three dimensions [2-4].

Since the linear Stokes vector depends on three real-valued parameters, one needs at least three intensity measurements to estimate it. However, many experimental schemes use a larger number of measurements. This strategy is generally thought to improve the estimation precision [5], but it may increase the complexity of the system and the measurement time. Our goal in this paper will be to investigate the influence of the number of measurements on the estimation precision of the linear Stokes vector, the DOLP, and the AOP. We shall see that this influence depends on the dominant type of perturbation that affects the measurements. We will 
consider three different types of perturbations: additive noise that is independent of the measured intensity, Poisson shot noise, and noise due to imperfect knowledge of the parameters of the measurement system. It should be noted that these issues have already been addressed by many authors in the more complex cases of full Stokes and Mueller measurements [5-13]. Restricting ourselves to the case of linear Stokes measurements will make it possible to obtain closed-form solutions for the estimation variances that clearly exhibit the influence of the number of measurements.

\section{Position of the Problem}

We assume that the polarization state of the light is defined by the three components of the linear Stokes vector $\mathbf{S}=\left(S_{0}, S_{1}, S_{2}\right)^{T}$, where $T$ denotes transposition. It can also be written as [1]

$$
\mathbf{S}=S_{0}(1, P \cos 2 \alpha, P \sin 2 \alpha)^{T},
$$

where $S_{0}$ represents the intensity of light, $P \in[0,1]$ is the DOLP, and $\alpha \in\left[-90^{\circ},+90^{\circ}\right]$ is the AOP, that is, the angle that the principal polarization state makes with a reference axis. To estimate the linear Stokes vector, one measures the light intensity at the output of a set of $N$ linear analyzers oriented with angles $\theta_{i}, i \in[1, N]$. Depending on the experimental setup, these $N$ measurements may be performed by $N$ different static devices or by the same device that is rotated $N$ times, mechanically or with help of an electro-optic device. The first type of measurement system can be called "division of wavefront" and the second "division of time" [14]. In both cases, the amount of light available for each measurement decreases with the number $N$. To take this into account, we will assume that the total amount of light (the number of photons) available is fixed, whatever the number of measurements. We also assume that the vector $\mathbf{S}$ that we want to estimate is the Stokes vector, whose intensity is equal to this total number of photons. Consequently, if $N$ measurements are performed, each measurement is made with this total amount of photons divided by $N$. Notice that we assume, for the sake of simplicity, that each of the $N$ measurements has the same duration and collects the same amount of light flux. Whatever the type of measurement system, in the absence of perturbation, the output intensity from the $i$ th measurement can be written as

$$
I_{i}=\frac{1}{2 N}\left[S_{0}+S_{1} \cos 2 \theta_{i}+S_{2} \sin 2 \theta_{i}\right],
$$

where $\theta_{i}$ are the angles of the analyzers. In Eq. (2), the fact that the measured intensity is inversely proportional to $N$ relies on the assumption that whatever the value of $N$, the number of photons gathered during one measurement is not sufficient to fill up the well of the CCD sensor. In other words, Eq. (2) is valid only in the case where the measurement is photon limited, and we will consider only this situation in this paper.

By using Eq. (1), it is easily seen that the intensity can also be written as

$$
I_{i}=\frac{S_{0}}{2 N}\left[1+P \cos \left(2 \theta_{i}-2 \alpha\right)\right] .
$$

Estimating $P$ and $\alpha$ thus corresponds to estimating the contrast and the phase of a sinusoid sampled on $N$ points. To perform this estimation, we gather the intensities $I_{i}$ into a vector:

$$
\mathbf{I}=\frac{1}{N} W \mathbf{S}
$$

with $W$ having the following $N \times 3$ matrix:

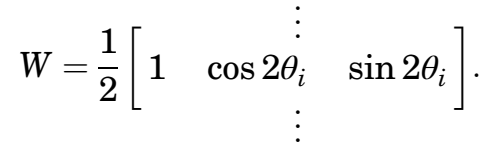

In practice, however, Eq. (4) does not hold strictly since $I$ is affected by noise and perturbations: it is thus a random vector. Our goal will be to estimate $\mathbf{S}$ from the random vector $\mathbf{I}$ in the presence of such perturbations. For that purpose, we will use the pseudoinverse estimator [15], which has the following expression:

$$
\hat{\mathbf{S}}=N W^{\dagger} \mathbf{I}
$$

with

$$
W^{\dagger}=\left(W^{T} W\right)^{-1} W^{T} .
$$

This estimator is optimal in the maximum likelihood sense when the noise that perturbs the measurement vector $\mathbf{I}$ is additive, white, and Gaussian [15]. For other types of noise, it may not be optimal but it is a simple and efficient estimator that we shall use throughout this paper. If we assume that the noise affecting the measurements is such that $\langle\mathbf{I}\rangle=1 / N \times$ $W S$, where $\langle$.$\rangle denotes ensemble averaging, the pseu-$ doinverse estimator defined in Eq. (6) is unbiased since $\langle\hat{\mathbf{S}}\rangle=\mathbf{S}$. This result is independent of the number of measurements $N$. Consequently, the estimation performance will depend only on the variance of $\hat{\mathbf{S}}$.

Once the Stokes vector has been estimated, the DOLP is estimated by

$$
\hat{P}=\frac{\sqrt{\hat{S}_{1}^{2}+\hat{S}_{2}^{2}}}{\hat{S}_{0}},
$$

and the $\mathrm{AOP} \alpha$ by

$$
\hat{\alpha}=\frac{1}{2} \arg \left[\hat{S}_{1}+i \hat{S}_{2}\right],
$$


where $\arg [$.$] denotes the argument (phase) of a$ complex number. In other words,

$$
\begin{aligned}
& \text { if } S_{1}>0: \hat{\alpha}=\frac{1}{2} \arctan \left[\begin{array}{l}
\hat{S}_{2} \\
\hat{S}_{1}
\end{array}\right], \\
& \text { if } S_{1}<0: \hat{\alpha}=\frac{\pi}{2} \times \operatorname{sgn}\left(S_{2}\right)+\frac{1}{2} \arctan \left[\frac{\hat{S}_{2}}{\hat{S}_{1}}\right],
\end{aligned}
$$

where $\operatorname{sgn}(x)=1$ if $x \geq 0,-1$ otherwise.

\section{Estimation in the Presence of Additive Noise}

We will first consider that the noise that perturbs the measurements, $\mathbf{I}$, is additive and Gaussian. In other words,

$$
\mathbf{I}=\frac{1}{N} W \mathbf{S}+\mathbf{b},
$$

where b is a Gaussian random vector that is assumed to be white with zero mean and variance $\sigma^{2}$. We shall analyze, in this section, the estimation precision of the Stokes vector, the DOLP, and the AOP. A similar approach has been used by Takakura and Ahmad to study estimation precision of Mueller matrices [12].

\section{A. Estimation of the Stokes Vector}

The estimation performance of the pseudoinverse estimator of the Stokes vector is determined by its covariance matrix, which, from Eq. (6), is equal to

$$
\Gamma^{\hat{\mathbf{S}}}=N^{2} W^{\dagger} \Gamma^{\mathbf{I}}\left(W^{\dagger}\right)^{T},
$$

where $\Gamma^{\mathrm{I}}$ is the covariance matrix of the measurement vector $\mathbf{I}$. If the noise is additive as defined in Eq. (11), one has $\Gamma^{\mathbf{I}}=\sigma^{2} \mathcal{I}_{N}$, where $\mathcal{I}_{N}$ denotes the $N \times \bar{N}$ identity matrix. The covariance matrix of the estimator is thus

$$
\Gamma^{\hat{\mathbf{S}}}=N^{2} \sigma^{2}\left(W^{T} W\right)^{-1},
$$

where we have made use of the expression of the pseudoinverse estimator in Eq. (7). The covariance matrix is a function of the measurement matrix $W$, which depends on the chosen measurement angles $\theta_{i}$. This choice constitutes a degree of freedom of the measurement system, which can be used to optimize its performance. A classical performance criterion is the trace of $\Gamma^{\hat{\mathbf{s}}}$, which represents the sum of the variances on the three components of the Stokes vector. It can be shown [16] that the angles that minimize this trace are such that

$$
\theta_{i}=\theta_{0}+\frac{(i-1) \times 180^{\circ}}{N},
$$

where $i$ varies from 1 to $N$. This corresponds to analyzer angles evenly distributed over the half-circle. In the following, we will choose this configuration with $\theta_{0}=0$, which means that the reference axis used to define the Stokes vector is given by the orientation of the first analyzer. Using Eqs. (5) and (14), it is easily shown that

$$
\left(W^{T} W\right)^{-1}=\frac{4}{N}\left[\begin{array}{lll}
1 & 0 & 0 \\
0 & 2 & 0 \\
0 & 0 & 2
\end{array}\right],
$$

where we have used the following relations:

$$
\begin{gathered}
\sum_{i=1}^{N} \cos 2 \theta_{i}=\sum_{i=1}^{N} \sin 2 \theta_{i}=\sum_{i=1}^{N} \cos 2 \theta_{i} \sin 2 \theta_{i}=0 \\
\sum_{i=1}^{N} \cos ^{2} 2 \theta_{i}=\sum_{i=1}^{N} \sin ^{2} 2 \theta_{i}=\frac{N}{2}
\end{gathered}
$$

that are valid if the values of $\theta_{i}$ verify Eq. (14). Substituting Eqs. (5) and (15) in Eq. (7), we find that the pseudoinverse matrix has the following expression:

$$
W^{\dagger}=\frac{2}{N}\left[\begin{array}{ccc}
1 & \ldots & 1 \\
2 \cos 2 \theta_{1} & \ldots & 2 \cos 2 \theta_{N} \\
2 \sin 2 \theta_{1} & \ldots & 2 \sin 2 \theta_{N}
\end{array}\right] .
$$

Moreover, substituting Eq. (15) in Eq. (13), one obtains the covariance matrix of the estimator:

$$
\Gamma_{\hat{\mathbf{s}}}=4 N \sigma^{2}\left[\begin{array}{lll}
1 & 0 & 0 \\
0 & 2 & 0 \\
0 & 0 & 2
\end{array}\right] .
$$

This matrix is diagonal, which means that the fluctuations of the estimates of each component of the Stokes vector are uncorrelated. The estimation variances of the Stokes parameters are the diagonal values of this matrix. It is seen that the variances on $S_{1}$ and $S_{2}$ are twice that on $S_{0}$.

In order to study the variation of the covariance matrix with respect to the number of measurement $N$, one has to distinguish two cases, depending on whether the variance $\sigma^{2}$ of the noise depends or not on the time spent by each measure, which is proportional to $1 / N$. If $\sigma^{2}$ is independent of the measurement time, such as, for example, the readout noise of a CCD sensor, it is seen in Eq. (18) that the estimation variances of all Stokes parameters increase linearly with $N$. In other words, the estimation precision decreases with the number of measurements! This surprising result is, in fact, easy to understand. Indeed, each single measurement adds a constant amount of noise that is independent of the signal value, and thus by spreading the available amount of light over more measurements, one increases the amount of noise. Consequently, when signal and time-independent additive detector noise is dominant, it is preferable to use the smallest possible number of measurements, that is, three measurement angles at $\theta_{0}=0^{\circ}, 60^{\circ}$, and $120^{\circ}$. However, it may also happen that the variance of the noise is proportional to the measurement time, so that $\sigma^{2}=a_{0} / N$, such as dark current noise, for example [17]. In this case, Eq. (18) shows that the estimation variances of all 
Stokes parameters are independent of the number of measurements $N$.

B. Estimation of the Degree of Linear Polarization and the Angle of Polarization

Let us now turn to the estimation of the DOLP and the AOP. The estimators of these parameters are given in Eqs. () and (9). These are nonlinear functions of the Stokes vector, and it is thus difficult to obtain closed-form expressions of their variances. However, one can determine approximate values of these variances that are valid in the case of small perturbations. Indeed, let $\mathbf{X}$ be a $K$-dimensional random vector with mean $\langle\mathbf{X}\rangle$ and covariance matrix $\Gamma^{\mathbf{X}}$, and let $y=f(\mathbf{X})$ be a random variable that is a function of this vector. If the variations of $\mathbf{X}$ around $\langle\mathbf{X}\rangle$ are sufficiently small and the function $f$ is sufficiently "smooth" around $\langle\mathbf{X}\rangle$, then [18]

$$
\langle y\rangle \simeq f(\langle\mathbf{X}\rangle) \quad \text { and } \quad \operatorname{VAR}[y] \simeq[\nabla f(\langle\mathbf{X}\rangle)]^{T} \Gamma_{\mathbf{X}} \nabla f(\langle\mathbf{X}\rangle)
$$

where $\nabla f(\mathbf{x})=\left[\partial f / \partial X_{1}(\mathbf{x}), \ldots, \partial f / \partial X_{K}(\mathbf{x})\right]^{T}$ is the gradient of the function $f$. Keep in mind that it is only an approximation, relying on strong assumptions that are not always fulfilled in practice (see below). However, it is useful to get physical insight into the parameters that govern the estimation precision.

Let us first consider estimation of the DOLP. Using Eq. (8), the expression of its gradient is

$$
\nabla \hat{P}=\frac{1}{P S_{0}^{2}}\left[-P^{2} S_{0}, S_{1}, S_{2}\right]^{T} .
$$

The approximate variance of $\hat{P}$ is obtained by substituting Eqs. (20) and (18) into Eq. (19). Straightforward computations lead to

$$
\operatorname{VAR}[\hat{P}]=\left(\frac{\sigma}{S_{0}}\right)^{2} 4 N\left(2+P^{2}\right) .
$$

This expression shows that when $\sigma^{2}$ is independent of $N$, the variance of $\hat{P}$ increases linearly with $N$. On the other hand, when it is inversely proportional to $N$, the variance of $\hat{P}$ is independent of $N$. Moreover, this variance depends on the input polarization state only through its intensity $S_{0}$ and its DOLP. It is thus independent of the AOP. It is also inversely proportional to the square of the ratio $S_{0} / \sigma$, which can be considered as the intensity signal-to-noise ratio (SNR), and it increases with the actual value of $P$. It is interesting to note that preliminary experimental evidence of this phenomenon has been shown by Vaughn and Hoover in a Mueller polarimeter [5] .

It must be noticed that the function in Eq. (8), considered as a function of $\hat{\mathbf{S}}$, has a discontinuous first derivative at the point $(1,0,0)^{T}$. Consequently, the smoothness condition necessary for the validity of Eq. (21) is fulfilled only if $\hat{S}_{1}$ and $\hat{S}_{2}$ are sufficiently far from zero, that is, for sufficiently high values of $P$.
For example, if the light is completely depolarized, $\hat{S}_{1}$ or $S_{2}$ are Gaussian random variables with zero mean. If we neglect the fluctuations of $\hat{S}_{0}$, the random variable $\hat{P}$ has a Rayleigh probability density function whose variance is [18] $\left(\sigma / S_{0}\right)^{2} 8 N(2-\pi / 2)$, which is about half of the value given by Eq. (21). On the other hand, when $P$ is sufficiently large and the noise variance sufficiently low, the random variables $\hat{S}_{1}$ and $\hat{S}_{2}$ have a very low probability to reach zero and Eq. (21) is valid.

Let us now consider estimation of the AOP. From Eq. (9), the expression of its gradient is

$$
\nabla \hat{\alpha}=\frac{1}{2 P^{2} S_{0}^{2}}\left[0,-S_{2}, S_{1}\right]^{T} .
$$

The approximate variance of $\hat{\alpha}$ is obtained by substituting Eqs. (22) and (18) into Eq. (19). One obtains

$$
\operatorname{VAR}[\alpha]=\left(\frac{\sigma}{S_{0}}\right)^{2} \frac{2 N}{P^{2}} .
$$

It must be noted that this expression of the variance is valid when $\alpha$ is expressed in radian. The variance of $\hat{\alpha}$ is proportional to the number of measurements $N$ if $\sigma^{2}$ is independent of $N$ and inversely proportional to the intensity SNR. It is also inversely proportional to $P^{2}$, which means that the estimation precision of $\alpha$ gets worse as the light becomes less and less polarized. This is easily understandable, since as $P$ decreases, the polarized fraction of input light, which defines the AOP, decreases.

Here again, it must be noticed that Eq. (23) is valid only for sufficiently large values of $P$. In particular, it tends to infinity when $P$ tends to zero, whereas the maximal variance of $\hat{\alpha}$ is clearly finite. Indeed, the variance of $\hat{\alpha}$ is maximal when this random variable is uniformly distributed between $-90^{\circ}$ and $+90^{\circ}$. This happens, for example, when the light is totally depolarized $(P=0)$, so that $\hat{S}_{1}$ and $\hat{S}_{2}$ are zero-mean random variables with identical variances. It is well known [18] that in this case, Eq. (10) leads to a uniform probability density function for $\hat{\alpha}$. In conclusion, Eqs. (21) and (23) give good insight in the parameters that govern the estimation precision of the DOLP and the AOP but are valid only for sufficiently polarized light ( $P$ sufficiently high). Their actual domain of validity depends on the value of $P$ and of the SNR, $S_{0} / \sigma$. If they are not valid, one has to determine the actual probability density functions of $\hat{P}$ and $\hat{\alpha}$ from that of $\hat{S}$ and calculate the variances from them.

\section{Estimation in the Presence of Signal Dependent Poisson Shot Noise}

In many cases, the dominant source of noise in images is Poisson shot noise. The influence of this source of noise on the estimation of the full Stokes vector has been addressed by Sabatke et al. [8], Gamiz and Belsher [19], and Goudail [20]. In the presence of Poisson shot noise, $\mathbf{I}$ is a random vector such that [20] 


$$
\langle\mathbf{I}\rangle=\operatorname{VAR}[\mathbf{I}]=\frac{1}{N} W \mathbf{S},
$$

where VAR[.] denotes the variance of a random vector. From the properties of shot noise, the fluctuations are statistically independent from one intensity measurement to the other and the covariance matrix $\Gamma^{I}$ of I is, thus, diagonal. In this section, we will study the estimation precision of the Stokes vector, the DOLP, and the AOP in the presence of this type of noise.

\section{A. Estimation of the Stokes Parameters}

To estimate $\mathbf{S}$ from $\mathbf{I}$, we will use the pseudoinverse estimator. Please note that in the presence of Poisson noise, the maximum likelihood estimator has a different expression [21] and is not closed form. Since the pseudoinverse is a simple and closed-form algorithm that gives good results in practice, we have used it in this study. It has been shown in [20] that the covariance matrix of the Stokes vector can be written as

$$
\Gamma_{i j}^{\hat{S}}=N \sum_{k=0}^{2} S_{k} \sum_{n=1}^{N} W_{i n}^{\dagger} W_{j n}^{\dagger} W_{n k} .
$$

The expression of the covariance matrix depends on the value $N$ of the numbers of measurements. Indeed, for $N=3$, substituting Eqs. (5) and (7) into Eq. (25) leads to

$$
\Gamma^{\hat{S}}=2\left[\begin{array}{ccc}
S_{0} & S_{1} & S_{2} \\
S_{1} & 2 S_{0}+S_{1} & -S_{2} \\
S_{2} & -S_{2} & 2 S_{0}-S_{1}
\end{array}\right],
$$

and for $N \geq 4$ :

$$
\Gamma^{\hat{S}}=2\left[\begin{array}{ccc}
S_{0} & S_{1} & S_{2} \\
S_{1} & 2 S_{0} & 0 \\
S_{2} & 0 & 2 S_{0}
\end{array}\right] .
$$

To obtain these results, we have used Eq. (16) and the following results, which assume that the angles $\theta_{i}$ verify Eq. (14):

$$
\begin{gathered}
N \geq 3: \sum_{i=1}^{N} \cos ^{2} 2 \theta_{i} \sin 2 \theta_{i}=\sum_{i=1}^{N} \sin ^{3} 2 \theta_{i}=0, \\
N=3: \sum_{i=1}^{N} \cos ^{3} 2 \theta_{i}=\frac{3}{4}, \quad \sum_{i=1}^{N} \sin ^{2} 2 \theta_{i} \cos 2 \theta_{i}=-\frac{3}{4}, \\
N \geq 4: \sum_{i=1}^{N} \cos ^{3} 2 \theta_{i}=0, \quad \sum_{i=1}^{N} \sin ^{2} 2 \theta_{i} \cos 2 \theta_{i}=0 .
\end{gathered}
$$

It is seen in Eq. (27) that for $N \geq 4$, the covariance matrix does not depend on $N$. In the presence of Pois- son shot noise, there is no loss in terms of estimation precision when using more measurements, but there is no gain either. The same conclusion has been obtained by Sabatke et al. using simulations in the case of full Stokes vector estimation [8]. The variances $\gamma_{0}, \gamma_{1}$, and $\gamma_{2}$ of the three components of the linear Stokes vector are given by the diagonal values of the covariance matrix. For $N=3$, one has

$$
\left\{\begin{array}{l}
\gamma_{0}=2 S_{0} \\
\gamma_{1}=4 S_{0}+2 S_{1}=2 S_{0}(2+P \cos 2 \alpha) \\
\gamma_{2}=4 S_{0}-2 S_{1}=2 S_{0}(2-P \cos 2 \alpha)
\end{array} .\right.
$$

The estimation variances of $S_{1}$ and $S_{2}$ depend on the AOP $\alpha$ of the input state. However, the sum $\sum_{i=0}^{2} \gamma_{i}$ is independent of it, as predicted by theory [20]. On the other hand, for $N \geq 4$, one has

$$
\gamma_{0}=2 S_{0}, \quad \gamma_{1}=4 S_{0}, \quad \gamma_{2}=4 S_{0},
$$

and the variances on all components of the Stokes vector depend only on the intensity of the incident state, not on its DOLP nor on its AOP. This result is a justification for using four measurements instead of three, since it makes the estimation variance independent of the input polarization state. However, when Poisson shot noise is dominant, there is no justification for using a number of measurements larger than four.

B. Estimation of the Degree of Polarization and the Angle of Polarization

Let us now turn to the estimation precision of the DOLP and of the AOP. The approximate variance of $\hat{P}$ is obtained by substituting Eqs. (20) and (26) (for $N=3$ ) or (27) (for $N \geq 4$ ) into Eq. (19). This results in

$$
\begin{gathered}
\text { if } N=3, \quad \operatorname{VAR}[\hat{P}]=\frac{2}{S_{0}}\left[2-P^{2}+P \cos (6 \alpha)\right], \\
\quad \text { if } N \geq 4, \quad \operatorname{VAR}[\hat{P}]=\frac{2}{S_{0}}\left[2-P^{2}\right] .
\end{gathered}
$$

These expressions have the same restricted domain of validity, as specified in Section 2 . They thus require sufficiently large values of $P$. Figure 1 (a) illustrates the results obtained if this condition is fulfilled. We have chosen the following configuration: $S_{0}=2000$ photoelectrons, $P=0.7$, and we have plotted the variance of $\hat{P}$ for $\alpha$ varying between $-45^{\circ}$ and $+45^{\circ}$ and three values of $N=3,4$, and 8 . The solid curve with the markers corresponds to the theoretical formulas in Eqs. (33) and (34), and the dotted curve corresponds to the variance estimated by Monte Carlo simulations on $10^{4}$ trials.

When $N=3$, the variance of $\hat{P}$ is inversely proportional to $S_{0}$, which can be considered as the intensity SNR (ratio between the squared mean and the variance of the intensity) in the case of Poisson shot noise. 
It depends on the AOP $\alpha$. It has three local maxima for $\alpha=0^{\circ}, 60^{\circ}$, and $120^{\circ}$, that is, when the state of polarization is parallel to one of the analyzer directions, and three local minima when $\alpha=30^{\circ}, 90^{\circ}$, and $150^{\circ}$, in which cases, the AOP is orthogonal to one of the analyzers and in the middle of the two others. It is noticed that if the light is totally polarized $(P=1)$, the minimal variance is equal to zero. This surprising result comes from the fact that if the light is totally polarized, one measures an average of zero photoelectrons when the AOP is orthogonal to one of the analyzers. Since the noise has Poisson statistics, its variance is also zero, which leads to a perfect estimation. Of course, this is an ideal case. Taking into account a residual additive detector noise or the finite extinction degree of analyzers would lead to nonzero variance even in this case, which would, however, still correspond to a minimum.

When $N \geq 4$, the variance of $\hat{P}$ becomes independent of the number of measurements $N$ and of the AOP. It depends on the input state only through its intensity $S_{0}$ and its degree of polarization. It decreases with the actual value of $P$, contrary to the case of additive noise, where it increases with $P$ [see Eq. (21)]. It can be noted that when $N=3$, the estimation variance varies sinusoidally around the value that would be given by Eq. (34).

The approximate variance of the $\mathrm{AOP}$ is obtained by substituting Eqs. (22) and (26) (for $N=3$ ) or (27) (for $N \geq 4$ ) in Eq. (19). This results in

$$
\begin{gathered}
\text { if } N=3, \quad \operatorname{VAR}[\hat{\alpha}]=\frac{1}{P^{2} S_{0}}\left[1-\frac{P}{2} \cos (6 \alpha)\right], \\
\text { if } N \geq 4, \quad \operatorname{VAR}[\hat{\alpha}]=\frac{1}{P^{2} S_{0}} .
\end{gathered}
$$

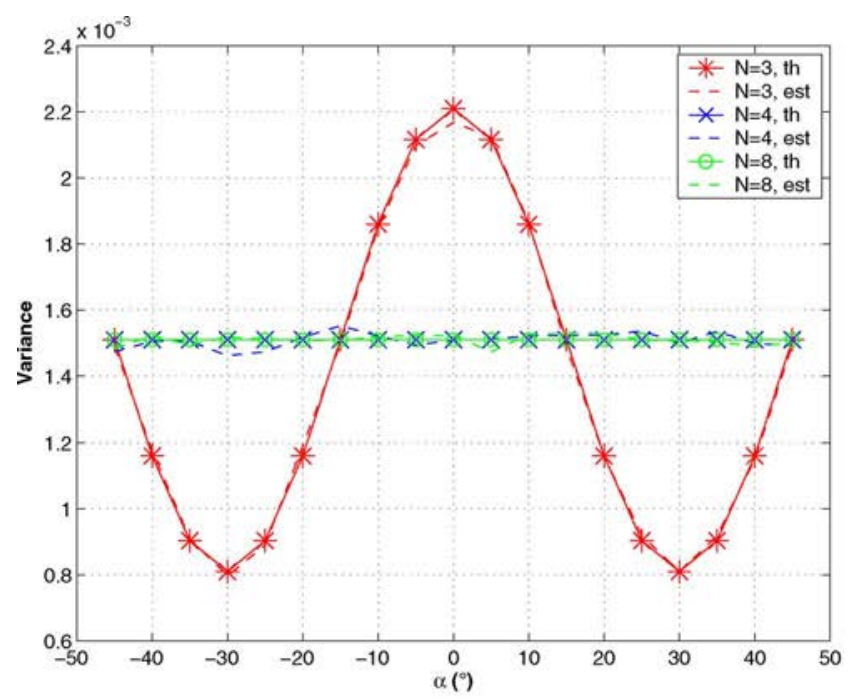

(a)
These results are illustrated in Fig. 1(b), which represents the estimation variance of $\alpha$ when it varies between $-45^{\circ}$ and $+45^{\circ}$ and three values of $N=3,4$, and 8 [the values of $S_{0}$ and $P$ are the same as in Fig. 1(a)]. The solid curve with the markers corresponds to the theoretical formulas in Eqs. (35) and (36) and the dotted curve to the variance estimated by Monte Carlo simulations on $10^{4}$ trials.

When $N=3$, the variance of $\hat{\alpha}$ depends on the actual value of $\alpha$. It is interesting to note that the maxima of variance of $\hat{\alpha}$ correspond to the minima of variance of $\hat{P}$ and vice versa. The minima of variance of $\hat{\alpha}$ correspond to cases where the input polarization state is parallel to one of the analyzers. It is also noticed that the variance of $\hat{\alpha}$ never reaches zero for any value of the AOP. When $N \geq 4$, the variance of $\hat{\alpha}$ is independent of the number of measurements $N$ and of the actual value of $\alpha$. As in the additive noise case, it is inversely proportional to the SNR and to $P^{2}$. Similarly to the DOLP case, it can be noted that when $N=3$, the variance of $\hat{\alpha}$ varies sinusoidally around the value that would be given by Eq. (36).

In conclusion, when Poisson shot noise is the dominant source of noise, it may be preferable to use four measurements instead of three, so that the estimation variances of the DOLP and of the AOP are independent of the actual value of the AOP. However, there is no justification for using more than four measurements, since the estimation variances are independent of $N$.

\section{Estimation in the Presence of Instrumental Noise}

In many cases, the dominant perturbations do not come from the detection process but from an imperfect knowledge of the characteristics of the measurement system. This problem has already been addressed in the case of full Stokes imagers [10] and of Mueller polarimeters $[\underline{5}, \underline{22}]$. In this paper, we restrict ourselves

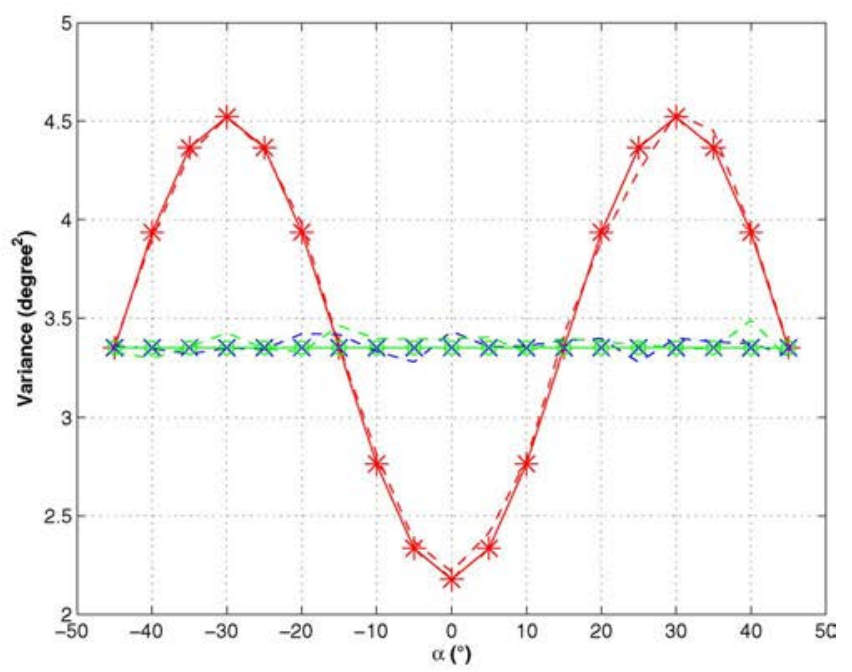

(b)

Fig. 1. (Color online) Theoretical and estimated variances of (a) the DOLP and (b) the AOP $\alpha$ as a function of the true value of the AOP in the presence of Poisson noise. $S_{0}=2000, P=0.7, \alpha \in\left[-45^{\circ},+45^{\circ}\right]$, and three values of $N=3,4$, and 8 are considered. 
to the case of linear Stokes images, but this will make it possible to obtain closed-form solutions for the estimation variances that clearly exhibit the influence of the number of measurements. We assume that the actual measurements are done with matrix $W$ defined in Eq. (5), whereas the estimation is made with a different matrix $W_{0}$ :

$$
\mathbf{I}=\frac{1}{N} W \mathbf{S} \quad \text { and } \quad \hat{\mathbf{S}}=N W_{0}^{\dagger} \mathbf{I} .
$$

In practice, $W_{0}$ will be the matrix determined by calibration, and the actual measurement matrix $W$ may deviate from it because of calibration noise or variations of the characteristics of the system after calibration (temperature changes or uncertainty on the analyzer angles due to mechanical movements). Note that to separate the problems, we assume that no other source of noise is present. The estimator of $\mathbf{S}$ can be written as

$$
\hat{\mathbf{S}}=\mathbf{S}+d M \mathbf{S},
$$

where the matrix $d M$ is defined as

$$
d M=W_{0}^{\dagger}\left(W-W_{0}\right) .
$$

The measurement matrix $W$ depends, in general, on an $N$-component parameter vector $\boldsymbol{\theta}$ (in our case, it is the set of analyzer angles) and will be written $W(\boldsymbol{\theta})$. The calibrated matrix is $W_{0}=W\left(\boldsymbol{\theta}_{0}\right)$, where $\boldsymbol{\theta}_{0}$ are the parameters estimated by calibration. If there is a small difference $d \boldsymbol{\theta}=\boldsymbol{\theta}-\boldsymbol{\theta}_{0}$, one can use a first-order development of $W$ with respect to $\theta$ and

$$
d M=\sum_{i=1}^{N} D_{i} d \theta_{i} \quad \text { with } D_{i}=W_{0}^{\dagger} \frac{\partial W}{\partial \theta_{i}} .
$$

\section{A. Estimation of the Stokes Vector}

Let us now assume that the parameter variation vector $d \boldsymbol{\theta}$ is a random vector with zero mean. In this case, the matrix $d M$ is random and so is the Stokes vector estimate $\hat{\mathbf{S}}$. Its mean is the true value $\mathbf{S}$, and its covariance matrix

$$
\Gamma^{\hat{\mathbf{s}}}=\left\langle d M d M^{T}\right\rangle=\sum_{i=1}^{N} \sum_{j=1}^{N} D_{i} \mathbf{S S}^{T} D_{j}^{T}\left\langle d \theta_{i} d \theta_{j}\right\rangle .
$$

To go further, one has to make assumptions on $d \boldsymbol{\theta}$. Let us assume that it is white, that is, $\left\langle d \theta_{i} d \theta_{j}\right\rangle=0$ if $i \neq j$ and $\sigma_{\theta}^{2}$ otherwise. In this case,

$$
\Gamma^{\hat{\mathbf{S}}}=\sigma_{\theta}^{2} \sum_{i=1}^{N} D_{i} \mathbf{S S}^{T} D_{i}^{T} .
$$

In the particular problem of linear Stokes vector estimation, the parameters of the measurement matrix are the angles $\theta_{i}$ of the analyzers. The matrices $D_{i}$ can be computed from Eqs. (5) and (7). The covariance matrix of the estimator is then found by substituting this result into Eq. (42). After cumbersome, but elementary, computations, one obtains the expressions of the covariance matrix that depend on the value of $N$ :

$$
\begin{aligned}
& \text { if } N=3, \quad \Gamma^{\hat{\mathrm{s}}}=\frac{2 \sigma_{\theta}^{2}}{3} \\
& \quad \times\left[\begin{array}{ccc}
P^{2} S_{0}^{2} & S_{2}^{2}-S_{1}^{2} & 2 S_{1} S_{2} \\
S_{2}^{2}-S_{1}^{2} & P^{2} S_{0}^{2}+2 S_{2}^{2} & -2 S_{1} S_{2} \\
2 S_{1} S_{2} & -2 S_{1} S_{2} & P^{2} S_{0}^{2}+2 S_{1}^{2}
\end{array}\right], \\
& \text { if } N=4, \quad \Gamma^{\hat{\mathrm{s}}}=\frac{\sigma_{\theta}^{2}}{2}\left[\begin{array}{ccc}
P^{2} S_{0}^{2} & 0 & 0 \\
0 & 4 S_{2}^{2} & 0 \\
0 & 0 & 4 S_{1}^{2}
\end{array}\right],
\end{aligned}
$$

if $N>4, \quad \Gamma^{\hat{\mathrm{s}}}=\frac{2 \sigma_{\theta}^{2}}{N}$

$$
\times\left[\begin{array}{ccc}
P^{2} S_{0}^{2} & 0 & 0 \\
0 & P^{2} S_{0}^{2}+2 S_{2}^{2} & -2 S_{1} S_{2} \\
0 & -2 S_{1} S_{2} & P^{2} S_{0}^{2}+2 S_{1}^{2}
\end{array}\right] .
$$

For obtaining these results, we have taken into account explicitly the fact that the nominal values $\theta_{i}$ are evenly distributed around the circle and that $\theta_{0}=0$ [Eq. (14)]. We have thus used Eqs. (16) and (30), and the following relations:

$$
N \geq 3: \sum_{i=1}^{N} \cos ^{3} 2 \theta_{i} \sin 2 \theta_{i}=\sum_{i=1}^{N} \cos 2 \theta_{i} \sin ^{3} 2 \theta_{i}=0,
$$

$$
\begin{aligned}
N= & 4: \sum_{i=1}^{N} \cos ^{4} 2 \theta_{i}=2, \quad \sum_{i=1}^{N} \sin ^{4} 2 \theta_{i}=2, \\
& \sum_{i=1}^{N} \cos ^{2} 2 \theta_{i} \sin ^{2} 2 \theta_{i}=0
\end{aligned}
$$

$$
\begin{aligned}
N \neq & 4: \sum_{i=1}^{N} \cos ^{4} 2 \theta_{i}=\frac{3 N}{8}, \quad \sum_{i=1}^{N} \sin ^{4} 2 \theta_{i}=\frac{3 N}{8}, \\
& \sum_{i=1}^{N} \cos ^{2} 2 \theta_{i} \sin ^{2} 2 \theta_{i}=\frac{N}{8} .
\end{aligned}
$$

It is seen in Eqs. (43)-(45) that the nature of the intercorrelations between the fluctuations of the Stokes components depends on the value of $N$. However, for all values of $N$, the sum of the variances of the three components of the Stokes vector is 
independent of the AOP and equal to

$$
\operatorname{trace}\left[\Gamma^{\hat{\mathbf{S}}}\right]=\frac{10}{N} \sigma_{\theta}^{2} P^{2} S_{0}^{2} .
$$

It depends on the degree of polarization and is proportional to the square of the intensity $S_{0}$ of the input state. More importantly, this variance is inversely proportional to $N$. This means that it is preferable to sample the circle with a number of polarization angles that is as large as possible. Recalling the interpretation of the measure of the Stokes vector as the estimation of the parameters of a sinusoid [see Eq. (3)], this means that sampling more finely the sinusoid reduces the sensitivity to the fluctuation of the positions of the sampling points.

It is also noted that in the case $N=4$, the matrix is diagonal, which means that there is no correlation between the fluctuations of the Stokes parameters. Furthermore, the case $N=3$ is very similar to $N>$ 4 except that extra nondiagonal terms are nonzero.

B. Estimation of the Degree of Polarization and the Angle of Polarization

Let us now turn to the estimation precision of the DOLP and of the AOP. Depending on the value of $N$, the approximate variance of $\hat{P}$ is obtained by substituting Eqs. (20) and (43), or (44) or (45) into Eq. (19). After some cumbersome, but elementary, computation, one obtains

$$
\text { if } N=3, \quad \operatorname{VAR}[\hat{P}]=\frac{2 \sigma_{\theta}^{2}}{3} P^{2}\left[1+P^{2}+2 P \cos (6 \alpha)\right]
$$

$$
\begin{aligned}
& \text { if } N=4, \quad \operatorname{VAR}[\hat{P}]=\frac{\sigma_{\theta}^{2}}{2} P^{2}\left[1+P^{2}-\cos (8 \alpha)\right], \\
& \text { if } N>4, \quad \operatorname{VAR}[\hat{P}]=\frac{2 \sigma_{\theta}^{2}}{N} P^{2}\left[1+P^{2}\right] .
\end{aligned}
$$

Figure 2(a) illustrates these results. It represents the estimation variance of the DOLP for $\alpha$ varying between $-45^{\circ}$ and $+45^{\circ}$, three values of $N=3,4$, and 8 , and the same values of $S_{0}$ and $P$ as in Fig. 1 . The solid curve with the markers corresponds to the theoretical formulas in Eqs. (50) -(52), and the dotted curve corresponds to the variance estimated by Monte Carlo simulations on $10^{4}$ trials.

For $N=3$, the variance of $\hat{P}$ varies sinusoidally and reaches maxima for $\alpha=0^{\circ}, 60^{\circ}$, and $120^{\circ}$, that is, when the AOP of the incident state is parallel to one of the analyzers. For $N=4$, the variance also varies sinusoidally and reaches minima for $\alpha=$ $k \times 45^{\circ}$ ( $k$ is an integer), that is, when the AOP of the incident state is parallel to one of the analyzers. For $N>4$, it is independent of the AOP. One can note that when $N=3$ or 4 , the variance varies sinusoidally around the value that would be given by Eq. (52).
Globally, it is seen that the variance of $\hat{P}$ decreases with $N$.

The approximate variance of the AOP is obtained by substituting Eqs. (22), (43), and (4) or (45) into Eq. (19). This results in

$$
\begin{gathered}
\text { if } N=4, \quad \operatorname{VAR}[\hat{\alpha}]=\frac{\sigma_{\theta}^{2}}{8}[3+\cos (8 \alpha)], \\
\text { if } N \neq 4, \quad \operatorname{VAR}[\hat{\alpha}]=\frac{3}{2} \frac{\sigma_{\theta}^{2}}{N} .
\end{gathered}
$$

Figure 2(b) corresponds to the same values of $S_{0}$ and $P$ as in Fig. 2(a). It represents the estimation variance of the $\overline{\mathrm{AOP}}$ for $\alpha$ varying between $-45^{\circ}$ and $+45^{\circ}$ and three values of $N=3,4$, and 8 . The solid curve with the markers corresponds to the theoretical formulas in Eqs. (53) and (54), and the dotted curve corresponds to the variance estimated by Monte Carlo simulations on $10^{4}$ trials.

For $N=4$, the variance varies sinusoidally with the AOP and reaches maxima for $\alpha=k \times 45^{\circ}$, where $k$ is an integer. One can note that this is the inverse of what happens for the DOLP estimation. Here again, the variance varies around the value that would be given by Eq. (54). For $N=3$ and $N>4$, the variance is independent on the AOP. It decreases with $N$ and depends only on the variance of the analyzer angles. Interestingly, it is independent of $P$ for all values of $N$.

In conclusion, the variances on the DOLP and the AOP are both inversely proportional to $N$. This means that to minimize the influence of analyzer angle uncertainty, it is preferable to use a number of analyzer angles that is as large as possible.

\section{Discussion}

This paper was intended to answer a practical question: what is the number $N$ of polarization measurements that ensures optimal estimation precision of polarimetric parameters when the time (or total flux) available to do the measurement is fixed? We have seen that the answer depends on which source of noise dominantly affects the measurements. If the noise is additive and independent of the measurement time, it is better to use the minimal number of measurements. If Poisson shot noise is dominant, the estimation precision depends on the AOP if $N=$ 3 and is independent of it and of $N$ for $N \geq 4$. A good choice may thus be four measurements, since there is no justification for using a larger number of measurements. If the dominant noise is due to fluctuations of the analyzer angles, the estimation variance decreases with the number of measurements and provides a justification for using as many different analyzer angles as made possible by technology and the cost of the system. If the system is affected by another type of instrumental noise, the covariance matrix can still be computed from Eq. (42) but the conclusion, in terms of optimal number of measure- 


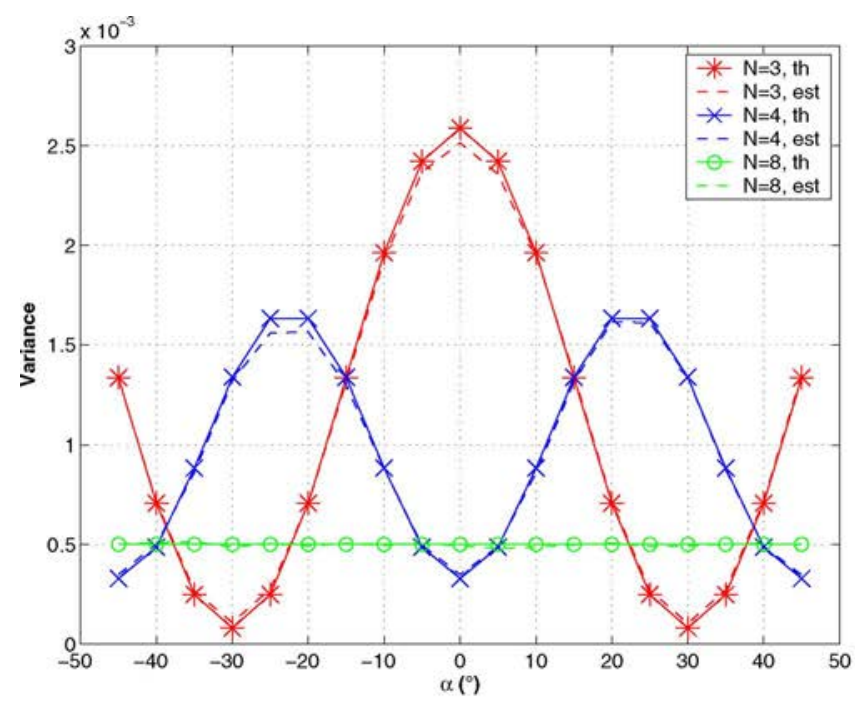

(a)

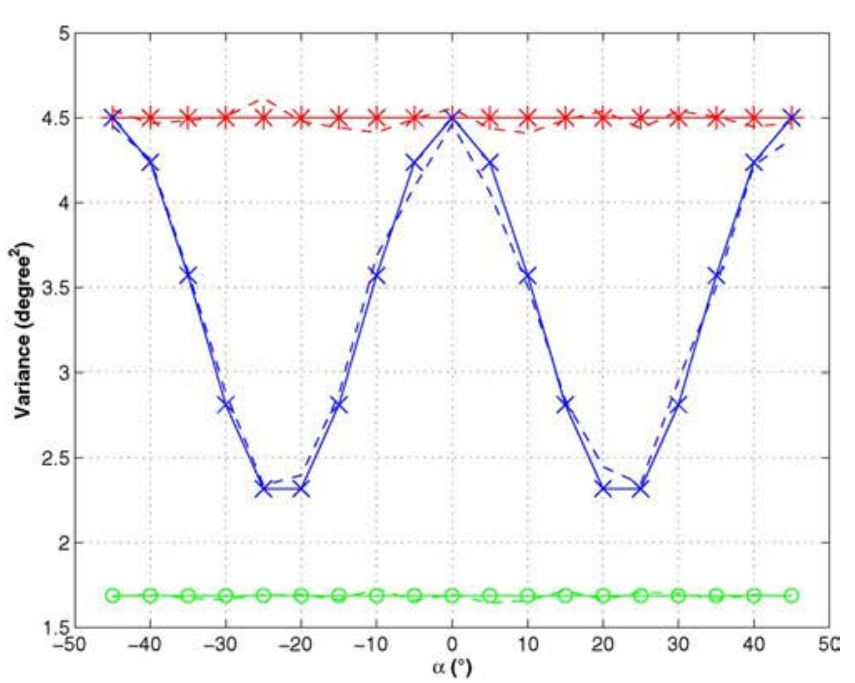

(b)

Fig. 2. (Color online) Theoretical and estimated variances of (a) the DOLP and (b) the AOP $\alpha$ as a function of the true value of the AOP in the presence of random fluctuations of the analyzer angles. $S_{0}=2000, P=0.7, \alpha \in\left[-45^{\circ},+45^{\circ}\right], \sigma_{\theta}=3^{\circ}$, and three values of $N=3,4$, and 8 are considered.

ments, may, of course, be different. Next, we address several applications and developments of the obtained results.

\section{A. Simultaneous Presence of Different Sources of Noise}

If the different considered types of noise are simultaneously present and have comparable orders of magnitude, they interact in a complex way. However, if they are statistically independent and sufficiently small with respect to the intensity measurement, their covariances (for Stokes measurements) and their variances (for estimation of the DOLP and the AOP) just add up. In this case, it is possible to determine the optimal number of measurements with the formulas determined in this paper. For example, assume that the three above-mentioned sources of noise are present. If we want the estimation of the DOLP and of the AOP to be invariant with respect to the AOP, we have to choose $N \geq 5$. We assume that the total variance can be calculated as the sum of the approximate variances associated to each type of noise. Let us first consider estimation of the DOLP. Using Eqs. (21), (34), and (52), one has

$$
\begin{aligned}
\operatorname{VAR}[\hat{P}](N)= & \frac{4\left(2+P^{2}\right)}{\mathrm{SNR}^{2}} N+\frac{2}{S_{0}}\left(2-P^{2}\right) \\
& +\frac{2 \sigma_{\theta}^{2} P^{2}\left(1+P^{2}\right)}{N},
\end{aligned}
$$

where $\mathrm{SNR}=S_{0} / \sigma$ is the intensity SNR relative to additive noise (we assume that $\sigma$ is independent of $N$ ). If we set to zero the derivative of this function with respect to $N$, we obtain the value of $N$ that minimizes the variance:

$$
N_{\min }^{P}=\sigma_{\theta} \mathrm{SNR} \sqrt{\frac{P^{2}\left(1+P^{2}\right)}{2\left(2+P^{2}\right)}} .
$$

The same reasoning with the AOP leads to the value of $N$ that minimizes the estimation variance of the AOP:

$$
N_{\min }^{\alpha}=\sigma_{\theta} \mathrm{SNR} \frac{\sqrt{3}}{2} P
$$

For example, let us assume that $\mathrm{SNR}=500, \sigma_{\theta}=1^{\circ}$, and $P=0.5$. One obtains $N_{\min }^{P}=2.3$ and $N_{\min }^{\alpha}=3.8$. One notices that the optimal values of $N$ for estimation of $P$ and $\alpha$ are different. In this case, both are inferior to 5 , and the configuration $N=5$ is thus optimal if one wishes the estimation variance of the DOLP and of the AOP to be independent of the AOP. Any larger value of $N$ would lead to higher estimation variance. Let us now assume that the analysis angles are less precisely known, so that $\sigma_{\theta}=3^{\circ}$. Equations (56) and (57) lead to $N_{\min }^{P}=6.9$ and $N_{\min }^{\alpha}=11.3$. Depending on the parameter that requires the highest precision, one will thus choose $N$ between 7 and 11 . Of course, other criteria, such as technological feasibility, power consumption, or cost, will also have to be taken into account.

\section{B. Introduction of a Penalty Due to Multiple Measurements}

Until now, we have made the assumption that the same total flux is collected, whatever the number $N$ of measurements. This assumption may not be valid. For example, in a division of time polarizer, making a large number of measurements may cause a significant penalty in lost integration time. Likewise, in the division of wavefront polarimeters, each division is usually accompanied by the same loss of efficiency. In order to take this into account, we can assume that at each measurement, a fraction $\varepsilon$ of the incoming flux is lost. Consequently, the vector of measured intensities [Eq. (4)] becomes 


$$
\mathbf{I}=\left(\frac{1}{N}-\varepsilon\right) W \mathbf{S}
$$

and thus the unbiased estimate of the Stokes vector is

$$
\hat{\mathbf{S}}=\frac{N}{1-N \varepsilon} W^{\dagger} \mathbf{I} .
$$

Let us first consider the case of additive Gaussian noise. It is easily shown that the covariance of the estimated Stokes vector [see Eq. (12)] becomes

$$
\Gamma^{\hat{\mathbf{s}}}=\left(\frac{N}{1-N \varepsilon}\right)^{2} W^{\dagger} \Gamma^{\mathbf{I}}\left(W^{\dagger}\right)^{T}=\frac{N^{2}}{(1-N \varepsilon)^{2}} \sigma^{2}\left(W^{T} W\right)^{-1} .
$$

This expression has to be compared to Eq. (13), which corresponds to the case $\varepsilon=0$. Taking into account a nonzero value of $\varepsilon$ thus induces an increase of the variance of the estimated Stokes vector [Eq. (18)] by a factor $1 /(1-N \varepsilon)^{2}$, which is always larger than 1. The approximated variances of the DOLP [Eq. (21)] and of the AOP [Eq. (23)] are also multiplied by this factor.

Let us now consider Poisson shot noise. Taking into account Eq. (58), the covariance matrix of $\mathbf{I}$ is

$$
\Gamma_{i j}^{\mathbf{I}}=\left(\frac{1}{N}-\varepsilon\right) \sum_{k=0}^{2} W_{i k} S_{k} \quad \text { if } i=j, 0, \text { otherwise }
$$

and thus Eq. (60) leads to

$$
\Gamma_{i j}^{\hat{S}}=\frac{N}{1-N \varepsilon} \sum_{k=0}^{2} S_{k} \sum_{n=1}^{N} W_{i n}^{\dagger} W_{j n}^{\dagger} W_{n k} .
$$

This expression has to be compared to Eq. (25). We observe that taking into account a nonzero value of $\varepsilon$ induces a multiplication of the estimation variance of the Stokes vector [Eqs. (26) and (27)], the DOLP [Eqs. (33) and (34)], and the AOP [Eqs. (35) and (36)] by a factor $1 \overline{(1}-N \varepsilon)$.

Finally, it is easily seen that the penalty $\varepsilon$ has no influence in the case of instrumental noise due to approximate knowledge of analyzer directions. As a consequence, in the case where the three considered sources of noise are simultaneously present, the approximate variance of $\hat{P}$ becomes

$$
\begin{aligned}
\operatorname{VAR}[\hat{P}](N)= & \frac{4\left(2+P^{2}\right) N}{\operatorname{SNR}^{2}(1-N \varepsilon)^{2}}+\frac{2\left(2-P^{2}\right)}{S_{0}(1-N \varepsilon)} \\
& +\frac{2 \sigma_{\theta}^{2} P^{2}\left(1+P^{2}\right)}{N} .
\end{aligned}
$$

This equation has to be compared to Eq. (55). If we take the same numerical example as in Subsection $\underline{5 . \mathrm{A}}, \sigma_{\theta}=3^{\circ}$ and $\varepsilon=0.05$, the value of $N$, which corresponds to the minimal variance of $P$, is $N_{\min }^{P}=4$ and that which corresponds to minimal variance of $\alpha$ is $N_{\min }^{\alpha}=5$. These values are lower than those obtained for $\varepsilon=0$, which is normal since a penalty for each measurement has been enforced.

\section{Consequences of the Different Sources of Noise on Image Quality}

One has to pay attention to the fact that the abovementioned sources of noise manifest themselves in different ways in an image. Assume, for example, that we acquire a polarimetric image of a region with uniform polarimetric properties. The additive noise and the Poisson shot noise will result in fluctuations of the estimated polarimetric properties from one pixel to the next. On the other hand, the manifestation of the instrumental noise depends on the design of the polarimetric imager. If the imager performs local analysis of the polarization at the pixel level, such as in a microgrid polarimetric setup [23,24], a fluctuation of the polarization angle or of any other instrumental characteristic will also appear as a pixelwise fluctuation. If polarization modulation is performed by rotating analyzers that modulate the whole field of the image, the fluctuation of the polarimetric properties will be the same for all pixels of the image. This noise will thus appear as a bias common to all pixels of the image. The impact of these different types of fluctuations depends on the application. If absolute measurement of the polarimetric parameters is necessary, such as, for example, in astronomical measurements [25] or three-dimensional reconstruction $[3,4]$, the bias is an important factor. However, if the application is detection of a target whose polarimetric properties are different from those of the background, the pixelwise fluctuations due to additive and shot noise are more annoying than a bias common to all pixels of the image.

\section{Conclusion}

We have studied the influence of different sources of noise on the estimation precision of the degree of linear polarization and the angle of polarization as a function of the number $N$ of polarimetric measurements for a fixed incoming light flux. We have shown that, for all the considered sources of noise, the estimation precision may depend on the angle of polarization of the incoming light when three or four measurements are performed, but becomes independent of it when $N \geq 5$. Moreover, the variation of the estimation variance with $N$ depends on the type of noise: it increases with $N$ when the noise is additive; it is independent of $N$ in the presence of Poisson shot noise and decreases with $N$ when the angles of the analyzers fluctuate. In the general case, the optimal value of the number of measurements results from a compromise between the robustness to these different sources of noise.

This work has many perspectives. First, the influence of other types of instrumental sources of noise, such as the nonperfect and fluctuating extinction 
rate of analyzers, could be studied with the approach proposed in this paper. It would also be of great interest to extend the present analysis to full Stokes imagers that measure the four components of the Stokes vector. The mathematics are more complex, but we are confident that the conclusions, in terms of variation of the estimation precision with the number of measurements, will be similar to those presented in this paper.

The authors wish to thank their anonymous reviewers for fruitful suggestions that greatly helped improve the quality of this article.

\section{References}

1. S. Huard, "Polarized optical wave," in Polarization of Light (Wiley, 1997), pp. 1-35.

2. L. B. Wolff and T. E. Boult, "Constraining object features using a polarization reflectance model," IEEE Trans. Pattern Anal. Machine Intell. 13, 635-657 (1991).

3. D. Miyazaki, M. Kagesawa, and K. Ikeuchi, "Transparent surface modeling from a pair of polarization images," IEEE Trans. Pattern Anal. Machine Intell. 26, 73-82 (2004).

4. O. Morel, C. Stolz, F. Meriaudeau, and P. Gorria, "Active lighting applied to 3D reconstruction of specular metallic surfaces by polarization imaging," Appl. Opt. 45, 4062-4068 (2006).

5. I. J. Vaughn and B. G. Hoover, "Noise reduction in laser polarimeter based on discrete waveplate rotations," Opt. Express 16, 2091-2108 (2008).

6. D. H. Goldstein and R. A. Chipman, "Error analysis of a Mueller matrix polarimeter," J. Opt. Soc. Am. A 7, 693-700 (1990).

7. A. Ambirajan and D. C. Look, "Optimum angles for a polarimeter: part I," Opt. Eng. 34, 1651-1658 (1995).

8. D. S. Sabatke, M. R. Descour, E. L. Dereniak, W. C. Sweatt, S. A. Kemme, and G. S. Phipps, "Optimization of retardance for a complete Stokes polarimeter," Opt. Lett. 25, 802-804 (2000).

9. M. H. Smith, "Optimization of a dual-rotating-retarder Mueller matrix polarimeter," Appl. Opt. 41, 2488-2493 (2002).

10. J. S. Tyo, "Design of optimal polarimeters: maximization of the signal-to-noise ratio and minimization of systematic error," Appl. Opt. 41, 619-630 (2002).
11. J. Zallat, S. Ainouz, and M. P. Stoll, "Optimal configurations for imaging polarimeters: impact of image noise and systematic errors," J. Opt. A Pure Appl. Opt. 8, 807-814 (2006).

12. Y. Takakura and J. Elsayed Ahmad, "Noise distribution of Mueller matrices retrieved with active rotating polarimeters," Appl. Opt. 46, 7354-7364 (2007).

13. A. Ramos and M. Collados, "Error propagation in polarimetric demodulation," Appl. Opt. 47, 2541-2549 (2008).

14. J. S. Tyo, D. L. Goldstein, D. B. Chenault, and J. A. Shaw, "Review of passive imaging polarimetry for remote sensing applications," Appl. Opt. 45, 5453-5469 (2006).

15. S. M. Kay, Fundamentals of Statistical Signal ProcessingVolume I: Estimation Theory (Prentice-Hall, 1993).

16. J. S. Tyo, "Optimum linear combination strategy for an $N$ channel polarization sensitive imaging or vision system," J. Opt. Soc. Am. A 15, 359-366 (1998).

17. G. C. Holst, CCD Arrays, Cameras, and Displays, 2nd ed. (JCD, 1998).

18. A. Papoulis, Probability, Random Variables and Stochastic Processes (McGraw-Hill, 1991).

19. V. L. Gamiz and J. F. Belsher, "Performance limitations of a four channel polarimeter in the presence of detection noise," Opt. Eng. 41, 973-980 (2002).

20. F. Goudail, "Noise minimization and equalization for Stokes polarimeters in the presence of signal-dependent Poisson shot noise," Opt. Lett. 34, 647-649 (2009).

21. M. Unser and M. Eden, "Maximum likelihood estimation of linear signal parameters for Poisson processes," IEEE Trans. Acoust. Speech Signal Processing 36, 942-945 (1988).

22. K. M. Twietmeyer and R. Chipman, "Optimization of Mueller matrix polarimeters in the presence of error sources," Opt. Express 16, 11589-11603 (2008).

23. A. G. Andreau and Z. K. Kalyjian, "Polarization imaging: principles and integrated polarimeters," IEEE Sens. J. 2, 566-576 (2002)

24. D. L. Bowers, J. K. Boger, L. D. Wellems, S. E. Ortega, M. P. Fetrow, J. E. Hubbs, B. M. Black, B. M. Ratliff, and J. S. Tyo, "Unpolarized calibration and nonuniformity correction for LWIR microgrid imaging polarimeters," Opt. Eng. 47, 046403 (2008).

25. J. Hough, "Polarimetry: a powerful diagnostic tool in astronomy," Astron. Geophys. 47, 3.31-3.35 (2006). 